\title{
ANNE ILE ÇOCUK ARASINDAKI EŞSIZ BAĞ OLARAK BAĞLANMA; PRENATAL DÖNEMDEN ÇOCUKLUĞA
}

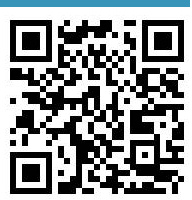

\author{
Damla Eyüboğlu'
}

1- Eskişehir Osmangazi Üniversitesi Tıp Fakültesi, Çocuk ve Ergen Ruh Sağlığı ve Hastalıkları AD. Eskişehir Türkiye

\begin{abstract}
Özet
Bu yazıda, bağlanma sürecinin prenatal dönemden başlayarak gözden geçirilmesi amaçlanmıştır. Prenatal dönemde annenin zihninden başlayan bağlanma süreci, doğum sonrası anne bebek ikilisinin etkileşimiyle kesinleşerek devam etmektedir. Anne bebek ilişkisinin niteliği ve bağlanma örüntüleri yaşam boyu bireyin hayatında izlerini sürer. Bağlanma, bireyin ileriki yaşamında ilişkilerine etki edeceği gibi, ruh sağlığı açısından da bağlanmanın güvenli veya güvensiz bağlanma olmasına göre koruyucu veya risk faktörü haline dönüşebilir. Bu nedenle, bağlanma sürecinin ve bu sürece etki eden faktörlerin anlaşılması, koruyucu ruh sağlığı ve halk sağığı açısından yol gösterici olacak, ayrıca yeni müdahale programlarının geliştirilmesine olanak sağlayacaktır.

Anahtar Kelimeler: Bağlanma, prenatal bağlanma, psikopatoloji.
\end{abstract}

\section{ATTACHMENT AS A UNIQUE BOND BETWEEN MOTHER AND CHILD; FROM PRENATAL PERIOD TO CHILDHOOD}

In this article, it is aimed to review the attachment process starting from the prenatal period. The process of attachment, which started from the mind of the mother in the prenatal period, continues by the interaction of the mother-baby as a couple after birth. The quality and attachment patterns of the mother-baby relationship last for a lifetime. Attachment will affect individuals' relationships in their life, and it can turn into a protective or risk factor depending on whether it is a secure or unsecure attachment in terms of mental health. Therefore, clarifying the attachment process and the factors affecting this process will be guide for the preventive mental health and public health, and will also allow the development of new intervention programs.

Keywords: Attachment, prenatal attachment, psychopathology.

Sorumlu Yazar / Corresponding Author: Dr. Öğr. Üyesi Damla Eyüboğlu

Eskişehir Osmangazi Üniversitesi Tıp Fakültesi, Çocuk ve Ergen Ruh Sağığı ve Hastalıkları AD.

Eskişehir, Türkiye.

e-posta / e-mail: deyuboglu@ogu.edu.tr

Geliş tarihi / Received : 08.04.2020, Kabul Tarihi / Accepted: 27.04.2020 
$\mathrm{B}$ ağlanma, Bowlby tarafından tanımlanan ve Ainsworth tarafından geliştirilen, çocuk ile birincil bakımveren arasındaki duygusal bağdır (1). Bebekle annesi arasında kurulan bu erken dönem ilişki çocuğun gelecek sosyal, duygusal ve bilişsel gelişimini etkilediği gibi yaşamın diğer yıllarında ruh sağlığı için de önemlidir $(2,3)$.

Anne bebek arasındaki ilişki bebeğin hem ruh sağlığı hem de fiziksel sağlığı hakkında önemli bir yordayıcıdır (4). Bowlby'e göre bebeğin zihinsel olarak sağlıklı büyüyebilmesi için bakımvereni ile her ikisinin de keyif aldığı sıcak, samimi ve sürekli bir ilişki içerisinde olmaları gerekir ve bu durum bağlanmanın temelini oluşturur (5). Lorenz ve Harlow'un 1958'de maymunlar üzerine yaptığı deneyde yavru maymunların çıplak telle kaplı anne maymun maketi tarafından beslendikten sonra havlu ile kaplı anne maymun maketine yöneldikleri ve orada vakit geçirme çabasında oldukları gözlenmiştir. Bu durum bebeğin temel intiyacının sadece beslenme olmadığı, yakınılı ve sıcaklığın da oldukça önemli olduğunu göstermiştir (6).

Normal koşullarda anneler, bebeğin doğumuyla birlikte sezgisel kapasitelerine başvurarak bebeğe bakım verebilmeye ve bebeğiyle senkronize ilişkiler kurmaya başlayabilmektedir (7). Erken dönemde, anne bebek ilişkisi bakışma, dokunma, ortak seslendirmeler ve karşılıklı duygusal ifadeler gibi sözel olmayan davranışlara dayanmaktadır $(8,9)$. Bebekler çok erken dönemden itibaren seslere, kendilerine gösterilen yüz ifadelerine ve bakışlara oldukça duyarlıdır. Anne de zihnini, dikkatini ve zamanını bebeğe vererek bebekten gelen sinyalleri anlayıp, uygun yanit vermeyi başarır ve bebek için "güvenli üs" haline gelir $(7,10)$. Güvenli üs varlığında bebekler gelişimsel dönemlerine uygun şekilde çevreyi keşfetmeye ve öğrenmeye başlar (10).

Bağlanma aşamalar halinde oluşur. Yaşamın ilk 8-12 haftasında bağlanmanın öncül davranışları kabul edilen emme, meme arama, anneye dönme davranışları gözlenir. $\mathrm{Bu}$ dönemde bebek henüz anneyi ayırt edemez. Bu dönemden 6 . aya kadar ise bebek anneyle daha uzun bakışmaya, gülümsemeye ve anneyi görünce ses çıkarmaya, sosyal tepkileri artmaya başlar. 24. aya kadar ise bağlanma netleşir ve bu dönemde bebek tüm intiyaçlarını karşılayan birincil bağlanma nesnesine yönelir (8).

\section{Erken Dönem Anne Bebek İlişkisini Etkileyen Faktörler ve Bağlanma Üzerine Etkileri}

Erken dönem anne bebek ilişkisini ve bağlanmayı etkileyen bazı içsel ve çevresel faktörler vardır. Annenin duyarlıı̆̆ı, postpartum sosyal destek, sosyoekonomik durum, annenin ruhsal durumu bu faktörler arasındadır. Anne duyarlıı̆̆; anne bebek etkileşiminde annenin ulaşılabilirliği, annenin sıcaklığı, kabulleniciliği, müdahaleci olmayan tavrı ve günlük bakım verirken bebeğin intiyacına hızlı yanıtı vericiliği olarak tanımlanmaktadır $(1,11)$. Duyarlı anneler, bebeğin değişen duygularını farkeder, onları yatıştııı ve bebeğin yaşadığı zor duygularla başedebilmesi için ona yol gösterir (12). Annelerin bebeklerinin duygularını anlaması ve bu duyguları yanıtlayıcılığı erken dönem anne bebek arasındaki güven ilişkisi için gereklidir (13). Ainsworth, annenin duyarlılığının annelik davranışını ideal biçimde yansıttığını belirtmiştir (1). Annenin duyarlıığının tatmin edici olup olmaması bebeğin mizacıyla ilişkilidir. Bazı bebekler için hafif bir uyarılma yeterli olurken, zor mizaçlı bebeklerin daha fazla uyarılmaya intiyacı olabilir. Bu nedenle bir bebeğe yönelik duyarlıık tatmin edici kabul 
edilirken başka bir bebek için intruziv bir anne tutumu olarak algılanabilir (14).

Doğumdan sonraki ilk aylarda annenin ruhsal durumunda bozulmalar olabilir. Postpartum depresyon ve kaygı düzeyi yüksek olan annelerin bebeği ile ilişkisinde bozulmalar olabilmektedir. Anne bebek etkileşiminde depresyon ve kaygının bakışma sürelerini ve karşılıklı keyif almayı azalttığı, aralarındaki senkronizasyonu bozduğu ve bu durumun bağlanma üzerine uzun vadede olumsuz etkiler bıraktığı vurgulanmaktadır (15-17).

Postpartum depresyon (PPD) birçok kültürde benzer oranlarda görülmektedir. PPD her yıl \%6.9-20 arasında görülmektedir $(18,19)$. PPD annenin bebeğine bakım verme niteliğine ve anne bebek etkileşimine olumsuz etki etmektedir. Feldman ve ark. (2007) annede depresyon şiddetlendikçe oksitosin düzeylerinde azalma olduğunu tespit ederek depresyonun bağlanma üzerine nörobiyolojik düzeyde etki ettiğini vurgulamışlardır (20). Anksiyete düzeyi yüksek annelerin de bebeklerine duyarlılığının azaldığı, bebeklerinden gelen sinyalleri anlamakta zorlandığı ve yanıtlayıcılıklarında fazla girici oldukları gözlenmiştir (16, 21-23).

\section{Bağlanma Örüntüleri}

Ainsworth ve ark. (1978) "Yabancı Durum" olarak bilinen ve çocuğun, annenin varlığında ve yokluğunda yabancıya ve anne ile ayrılıp birleşmeye gösterdiği reaksiyonların gözlemlendiği aşamalardan oluşan deneysel bir yöntem geliştirmişlerdir (32). Bu yönteme göre;

Güvenli bağlanma (Tip B) ilişkisi içerisinde olan çocuklar anne ile odada yalnız olduklarında kendilerini rahat hissederler ve etrafı keşfedebilirler. Fakat odaya bir yabancı girdiğinde oyun davranışı azalır. Bu çocuklar annelerinden ayrıldıklarında biraz huzursuzluk göstermekle birlikte anneleri geri dönünce ona karşı olumlu tepkiler vermekte ve kısa süre içerisinde oyun alanına geri dönüp çevreyi keşfetmeye
Sosyoekonomik durumun çocuk gelişimine etkileri üzerine yapılmış birçok çalışma mevcuttur. Düşük sosyoekonomik durumun ebeveynlerin yaşamında günlük stresi ve ebeveynlerin ruhsal sorunlarını arttırması nedeniyle ebeveyn çocuk etkileşimi üzerine olumsuz etki ettiği vurgulanmaktadır (24). Düşük sosyoekonomik düzey ile duyarlı ebeveyn davranışları ve güvenli bağlanma arasında negatif ilişki olduğu saptanmıştır $(25,26)$.

Anne bebek bağlanmasında etkili olduğu bilinen ancak daha fazla bilimsel araştırmaya intiyaç duyulan konulardan bir tanesi de annenin kendi bağlanma örüntüsü ve ebeveynleriyle ilişkisidir. Literatürde kendi annesiyle olumlu ilişkileri olan annelerin bebekleriyle erken dönemde daha olumlu etkileşimde bulunduğunu gösteren çalışmalar mevcuttur (27-29). Ek olarak kaygılı ve kaçıngan bağlanma örüntüsüne sahip bireylerin daha fazla ebeveynlik stresi ve depresyon deneyimledikleri belirtilmektedir (30,31). Ancak bu çalışmaların çoğunluğu annenin kendi ebeveyniyle olan ilişkisini özbildirim şeklinde değerlendirmiştir. Bağlanmanın kuşaklararası aktarımı konusunda uzun izlem çalışmalarına intiyaç vardır.

devam etmektedirler. Güvenli bağlanmanın gelişmesi için de çocuğun kesintisiz, tutarlı tepki veren, duyarlı ve her zaman ulaşılabilir bir anneye sahip olması gerekir $(1,32,33)$.

Güvensiz- Kaçıngan (Avoidan)

Bağlanma örüntüsüne sahip çocuklar anneleri odadan ayrıldıklarında ağlamazlar, anneleri geri döndüğünde ise annelerine ilgisiz davranıp oyunlarına devam ederler ve odaya yabancı kişi girdiğinde fazla ilgi göstermezler. Anne ile çocuk yeniden birleştiğinde her ikisinde de pek mutluluk gözlenmez. Çocuk anneye gitmez veya anne çocuğa yönelirse çocuk annenin ona yaklaşmasını reddeder. Ainsworth'e göre Kaçıngan Bağlanma örüntüsüne sahip 
çocukların anneleri reddedici, inmalkâr ve bebeğin taleplerine öfke ile yanıt veren tutum sergilemektedirler (32).

\section{Güvensiz-}

(Anksiyöz/Ambivalan)

Kaygılı/Kararsız

Bağlanma

örüntüsüne sahip çocuklar ise anneleri odadan ayrıldığında aşırı ağlama gibi yoğun protesto sergilerler, anne odaya geri döndüğünde ise yatışmaları çok güçtür. Anne çocuğu yatıştırmaya çabalarsa çocuk daha çok öfkelenebilir veya anksiyete yaşayabilir. Bu çocuklar odadaki eşyalarla ilgilenmeyi bırakıp anneye yapışabilirler. Ainsworth, bu davranışı annenin bebeğe cevap verememesi veya tutarsız olması ve bebeğin intiyaçlarına duyarsızlığı ile ilişkilendirmiştir. Bu bebekler, intiyaçları olduğunda annelerinin yanıt vereceğinden ya da yardımcı olacağından emin olamayan bebeklerdir. Bu durum sıklıkla depresif ve introvert özellikleri olan annelerde görülür. Sonuç olarak bu çocuklar da çok fazla talepkar ancak tatmin olamayan çocuklar olurlar $(32,34)$.

Dezorganize/Dağınık Bağlanma örüntüsü ise Main ve Solomon tarafından tanımlanan üçüncü güvensiz bağlanma örüntüsüdür. Dezorganize bağlanma hem kaçıngan hem de ambivalan bağlanma örüntülerinin özelliklerini sargiler. Dezorganize bağlanma ilişkisi temelinde bu çocuklar daha korkulu/kaygılı görünüp daha agresif davranışlar sergilerler. Yabancı durum testi sırasında anneleri odadan ayrıldığında "dona kalma" davranışı gösterip, anne ile tekrar birleşme anındaki davranışları ise öngörülemez. Ebeveynlerinde majör psikopatoloji olan çocuklar bu bağlanma örüntüsüne sahip olabilmektedir $(35,36)$.

\section{Bağlanmanın Yordayıcısı Olarak "Prenatal Bağlanma"}

Birçok araştırmacı bağlanmanın prenatal dönemde başladığını belirtmiştir (37, 38). Muller (1996) prenatal bağlanmayı, anne ile doğmamış bebeği arasında kurulan eşsiz bir ilişki olarak tanımlamıştır (37). Bu kurama katkı sunacak şekilde, Winnicott (1956), gebeliğin son döneminde başlayıp doğumdan sonraki ilk aylarda da devam eden ve çok özel bir psikolojik durum olarak belirttiği "primer annelik meşguliyeti”ni tanımlamıştır (39). Doğum esnasında bebeğini kaybeden annelerin, bebekleriyle fiziksel herhangi bir temasları olmasa dahi yas reaksiyonu gösterdiğini gözlemleyen çalışmalar da prenatal bağlanma kuramını desteklemiştir (40). Lumley gebelik takibinde ultrasonu kullanarak fetüsün görsel imgesinin annenin bağlanmasına katkı sunduğunu ve gebelik ilerledikçe fetüsü gerçek bir birey olarak algılamasının arttığını belirtmiştir (41). Muller, annenin kendi annesiyle olan erken dönem ilişki deneyimlerinin, annenin arkadaş, eş ve aile ilişkilerini etkileyebileceği gibi gebelik sürecine uyumunu ve prenatal bağlanmasını da etkileyeceğini vurgulamıştır (37).

Prenatal bağlanma; Maternal Fetal Bağlanma ölçeği, Prenatal Bağlanma Envanteri, Maternal Antenatal Bağlanma Ölçeği, Prenatal Maternal Bağlanma ölçeği kullanılarak değerlendirilebilmektedir.

Prenatal bağlanma ile postnatal bağlanma arasında önemli bir korelasyon olduğu, prenatal bağlanma düzeyi yüksek olan kadınların doğum sonrası anne bebek etkileşimlerinin daha iyi olduğu ve bunun güçlü bir yordayıcısı olduğu bildirilmektedir $(37,42)$.

Prenatal bağlanma annenin gebelik süresince annenin ve fetüsün sağlığını olumlu etkileyebileceği, için çok önemlidir. Prenatal bağlanması yüksek olan annelerin gebelik takiplerini düzenli yaptırdığı, hem kendi hem de bebeklerinin sağlıklarına dikkat ettiği belirtilmektedir (43). Ayrıca prenatal bağlanması yüksek olan gebelerin doğum sonrası daha yüksek oranda güvenli bağlanma geliştirdiği ve çocukların daha iyi gelişim gösterdikleri 
raporlanmıştır (44). Van Bussel ve ark. (2010) prenatal bağlanmanın postnatal bağlanmanın güçlü bir yordayıcısı olduğunu saptamışlardır (45). Prenatal ve postnatal bağlanmayı şekillendiren faktörler örtüşmeyebileceğinden prenatal bağlanmanın postnatal bağlanmayı tam olarak yordayabileceği söylenemese de aralarında güçlü bir ilişki olduğu birçok çalışmada saptanmıştır $(37,42,44)$.

\section{Bağlanma Örüntüleri ve Psikopatoloji}

\begin{abstract}
Güvenli bağlanmanın önemi sağlıklı biyopsikososyal gelişim açısından ve etkilerinin ömür boyu sürmesi nedeniyle literatürde birçok defa vurgulanmıştır. Yaşamın ilk üç yılında görülen psikolojik problemlerin çocuğun primer bakımvereniyle arasındaki ilişki ile doğrudan ilgilidir. Bu nedenle mevcut belirtileri anlayabilmek için ilişkinin niteliği aydınlatılmalıdır ve bu prensip tedavinin temelidir (46). Bowlby, annelerinden
\end{abstract} ayrılan ve bakımevlerinde yaşayan çocukları yakından incelemiş ve çocukların ayrılığa huzursuzluk, annelerini arama, ağlama, başkaları tarafından yatıştırılma çabalarına ise direnç gösterme şeklinde reaksiyonları olduğunu raporlamıştır. Ek olarak ayrılık uzadığındaysa çocuk apati ve gelişim geriliğinin gözlendiği anaklitik depresyon yaşayabilmektedir (5). Bağlanma kuramı temelinde, bebeğin primer bakım vereniyle ilişkisinin kalitesi, bebeğin gelecek yıllarda kendisi ve diğerleriyle ilgili algılarının temelini oluşturan "Çalışan İçsel Modeller" in oluşturur $(5,33)$.

Kaçıngan ve anksiyöz/ambivalan bağlanma örüntüleri çocukluk dönemindeki duygusal ve davranışsal sorunlarla ve erişkinlikteki diğer psikopatolojilerle ilişkiliyken, güvenli bağlanma ise koruyucu faktör olarak belirtilmektedir (36, 47). Bağlanma figürleri çocuğun stres anında duygu regülasyonuna yardımcı olur, destekler ve çocuğun rahatlamasını sağlar. Güvensiz bağlanma örüntüsüne sahip çocukların duygu düzenlemelerine, rahatlamalarına ve gereksinimlerinin karşılanmasında ebeveynlerinin yetersiz kalmaları veya tutarsız olmaları nedeniyle duygusal ve davranışsal sorunlara yatkın hale gelirler (47). Güvenli bağlanan çocuklara kıyasla, güvensiz bağlanma örüntüsüne sahip çocukların sosyal ilişkileri daha zayıf, akran ilişkileri daha sorunlu ve duygu düzenleme güçlüklerinin daha fazla olduğu raporlanmıştır (48, 49). Kaçıngan ve anksiyöz/ambivalan bağlanma örüntüsüne sahip çocukların depresyon dahil daha fazla içselleştirme (depresyon, anksiyete, sosyal izolasyon ve içe çekilme) sorunlarına yatkındırlar $(50,51)$. Ek olarak kaçıngan ve anksiyöz bağlanması olan çocuklarda güvenli bağlananlara kıyasla daha fazla davranım sorunu görülmektedir. Madigan ve ark.'nın yaptığı bir metaanaliz çalışmasında güvensiz bağlanması olan çocukların güvenli bağlanması olan çocuklara kıyasla içselleştirme sorunlarının 2.9 kat, davranış sorunlarının ise 2.4 kat daha fazla görüldüğü raporlanmıştır (50). Dezorganize bağlanması olan çocuklarda güvenli bağlanması olan çocuklara göre içselleştirme sorunlarından ziyade, davranış sorunlarının daha fazla gözlendiği saptanmıştır $(47,51)$. Ayrıca dezorganize bağlanma ile dissosiyatif bozukluk arasında önemli bir ilişki olduğu belirtilmektedir (36).

Prenatal bağlanmanın da bebeğin nörogelişimiyle ve duygusal/davranışsal sorunlarıyla ilişkili olduğunu belirten çalışmalar vardır. Prenatal bağlanması yüksek olan annelerin bebeklerin bebeklik ve erken çocukluk döneminde nörogelişimsel açıdan daha iyi seyrettikleri, konuşma ve motor becerilerinin daha iyi olduğu, ayrıca duygusal ve davranışsal sorunlarının daha az olduğu saptanmıştır $(44,52,53)$. 


\section{$\underline{\text { Kaynaklar }}$}

1. Ainsworth MD. Infant-mother attachment. Am J Psychol. 1979; 34(10): 932-37.

2. Siddiqui A. \& Hagglof B. Does maternal prenatal attachment predict postnatal motherinfant interaction? Early Hum Dev. 2000; 59(1):13-25.

3. Kim P, Mayes L, Feldman R, et al. Early postpartum Parental preoccupation and positive parenting thoughts: relationship with parent-infant interaction. Infant Ment Health J. 2013; 34(2): 104-116.

4. Farrell AK, Simpson JA, Carlson EA, et al. The impact of stress at different life stages on physical health and the buffering effects of maternal sensitivity. Health psychol. 2017; 36(1):35-44.

5. Bowlby J. Attachment and Loss: Volume 1. 1969.

6. Harlow HW, Zimmerman RR. Affectional response in the infant monkey. Science. 1959; 130: 421-32.

7. Güvenir T. Bebeklik ve erken çocukluk döneminde "duygu düzenlemesi ve bozuklukları". N. Erol (Ed), Bebek ruh sağlı̆̆ı içinde. ANKARA: Ankara Üniversitesi Basım Evi. 2012

8. Isseri E, ve Bodur Ş. Bağlanma Bozuklukları. A. Akay ve E. S. Ercan (Eds), Çocuk ve Ergen Ruh Sağlığı ve Hastalıkları içinde. Ankara: HYB Basım Yayın. 2016

9. Tronick EZ. Emotions and emotional communication in infants. Am Psychologist, 1989; 44(2), 112-9.

10. Serim Demirgören $B$, ve Özbek A. Bağlanma ve Gelişimi. K. Karabekiroğlu (Ed), Bebek Ruh Sağlığı (0-4 yaş) Temel Kitabı içinde. Ankara: Hekimlerbirliği yayınları, 2012

11. Tamis-LeMonda CS, \& Baumwell L. Parental sensitivity in early development: Definition, methods, measurement, and generalizability. In Maternal sensitivity: A scientific foundation for practice. Nova Science Publishers. 2011

12. Landry SH, Smith KE, and Swank PR. Responsive parenting: Establishing early foundations for social communication and problem solving. Dev Psychol, 2006; 42, 62742.

13. Bailey H N, Redden E, Pederson D R, \& Moran G. Parental disavowal of relationship difficulties fosters the development of insecure attachment. Can J Behav Sci, 2016; 48, 49-59.

14. Hane A A, Feldstein $S$ ve Dernetz $V H$. The relation between coordinated interpersonal timing and maternal sensitivity in four-monthold infants. J Psycholinguis Res, 2003; 32(5), 525-39.

15. Granat A, Gadassi R, Gilboa-Schechtman E ve Feldman R. Maternal depression and anxiety, social synchrony, and infant regulation of negative and positive emotions. Emotion, 2017; 17(1), 11-27.

16. Beebe B, Steele $M$, Jaffe J, Buck KA, Chen $H$, Cohen $P$, ... ve Feldstein S. Maternal anxiety symptoms and mother - infant self - and interactive contingency. Infant Ment Health J, 2011; 32(2), 174-206.

17. Beebe B, Jaffe J, Buck K, Chen H, Cohen P, Feldstein $S$, ve Andrews $H$. Six - week postpartum maternal depressive symptoms and 4 - month mother-infant self - and interactive contingency. Infant Ment Health J, 2008; 29(5), 442-71.

18. American Psychological Association (2017). Postpartum depression. Retrieved from https://www.apa.org/pi/women/resources/repor ts/ postpartum - depression

19. Centers for Disease Control and Prevention (2017). Reproductive health: Depression among women. Retrieved from https://www.cdc.gov/

reproductivehealth/depression/index.htm

20. Feldman R, Weller A, Zagoory-Sharon $O$, ve Levine A. Evidence for a neuroendocrinological foundation of human affiliation: plasma oxytocin levels across pregnancy and the postpartum period predict mother-infant bonding. Psychol Sci, 2007; 18(11), 965-70.

21. Feldman $R$, Greenbaum $C W$, Mayes $L C$, ve Erlich SH. Change in mother-infant interactive behavior: Relations to change in the mother, the infant, and the social context. Infant Behav Dev, 1997; 20(2), 151-63.

22. Stein A, Craske MG, Lehtonen A, Harvey A, Savage-McGlynn E, Davies B, ... ve Counsell $N$. Maternal cognitions and mother- infant interaction in postnatal depression and generalized anxiety disorder. J Abnorm, 2012; 121, 795-809.

23. Mount KS, Crockenberg SC, Jó PSB, \& Wagar $J L$. Maternal and child correlates of anxiety in 21/2-year-old children. Infant Behav Dev, 2010; $33,567-78$.

24. Letourneau NL, Duffett-Leger L, Levac L, Watson B, \& Young-Morris C. Socioeconomic status and child development: A meta-analysis. J Emot Behav Disord, 2013; 21, 211-24.

25. Cyr C, Euser EM, Bakermans-Kranenburg MJ, \& Van ljzendoor MH. Attachment security and disorganization in maltreating and high-risk families: A series of meta-analyses. Dev Psychopathol, 2010; 22, 87-108.

26. Mesman J, van IJzendoorn MH, \& BakermansKranenburg MJ. Unequal in opportunity, equal in process: Parental sensitivity promotes positive child development in ethnic minority families. Child Dev Perspect, 2012; 6, 239-50.

27. Crugnola CR, Gazzott, Spinelli M, lerardi E, Caprin C, ve Albizzat A. Maternal attachment influences mother-infant styles of regulation and play with objects at nine months. Attach Hum Dev, 2013; 15(2), 107-131.

28. Madigan S, Bakermans-Kranenburg MJ, Van ljzendoorn MH, Moran G, Pederson DR, ve Benoit D. Unresolved states of mind, anomalous parental behavior, and disorganized 
attachment: $A$ review and meta-analysis of a transmission gap. Attach Hum Dev, 2006; 8(2), 89-111.

29. Verhage ML, Schuengel C, Madigan S, Fearon $R M$, Oosterman $M$, Cassibba $R, \ldots$ ve van IJzendoorn $\mathrm{MH}$. Narrowing the transmission gap: A synthesis of three decades of research on intergenerational transmission of attachment. Psychol Bull, 2016; 142(4), 337366.

30. Stern JA, Fraley RC, Jones JD, Gross JT, Shaver PR, ve Cassidy J. Developmental processes across the first two years of parenthood: Stability and change in adult attachment style. Dev Psychol, 2018; 54(5), 975-988.

31. Jones JD, Cassidy J, ve Shaver PR. Parents' self-reported attachment styles: $A$ review of links with parenting behaviors, emotions, and cognitions. Pers Soc Psychol Rev, 2015; 19(1), 44-76.

32. Ainsworth MDS, Blehar MC, Waters E, \& Wall S. Patterns of attachment: A psychological study of the Strange Situation. Hillsdale, NJ: Erlbaum Associates, Inc. 1978

33. Bowlby J. The Making and Breaking of Affectional Bonds. Tavistock Publish, London, 1979

34. Goldberg S, Muir R, ve Kerr J. (Eds.) Attachment theory: Social development, and clinical perspectives. London: Routledge. 2013

35. Main $M$, \& Solomon J. Procedures for identifying infants as disorganized/disoriented during the Ainsworth Strange Situation. In M. T. Greenberg, D. Cicchetti, \& E. M. Cummings (Eds.), Attachment in the preschool years: Theory, research, and intervention (p. 121160). University of Chicago Press. 1990

36. van ljzendoorn $M H$, Schuengel C, \& Bakermans-Kranenburg MJ. Disorganized attachment in early childhood: Meta-analysis of precursors, concomitants, and sequelae. Dev Psychopathol, 1999; 11(2), 225-249.

37. Muller ME. Prenatal and Postnatal Attachment: A Modest Correlation. J Obstet Gynecol Neonatal Nurs, 1996; 25(2): 161-166.

38. Grace J. Development of maternal-fetal attachment during pregnancy. Nurs Res, 1989; 38(4), 228-232.

39. Winnicott DW. Primary maternal preoccupation. In $P$. Mariotti (Ed.), The new library of psychoanalysis. The maternal lineage: Identification, desire and transgenerational issues (pp. 59-66). New York, NY, US: Routledge/Taylor \& Francis Group. 2012

40. Kennell JH, Slyter $H$, ve Klaus $M H$. The mourning response of parents to the death of a newborn infant. N Engl J Med, 1970; 283, 344 349 .
41. Lumley JM. Through a glass darkly: Ultrasound and prenatal bonding. Birth. 1980; 17:214-217.

42. Siddiqui $A$, Hagglof $B$, Eisemann M. Own memories of upbringing as a determinant of prenatal attachment in expectant women. $J$ Reprod Infant Psyc. 2000;18(1):67-74.

43. Alhusen JL, Gross $D$, Hayat MJ, Woods $A B$, Sharps $P W$. The influence of maternal-fetal attachment and health practices on neonatal outcomes in low-income, urban women. Res Nurs Health 2012; 35(2):112-120.

44. Alhusen JL, Hayat MJ, Gross D. A longitudinal study of maternal attachment and infant developmental outcomes. Arch Women Ment Health , 2013; 16(6):521-529

45. Van Bussel JCH, Spitz B, Demyttenaere $K$. Reliability and validity of the Dutch version of the maternal antenatal attachment scale. Arch Womens Ment Health, 2010; 13(3):267-277

46. Zeanah $\mathrm{CH}$, Boris NW, Larrieu JA. Infant Development And Developmental Risk: $A$ Review Of The Past 10 Years. J Am Acad Child Adolesc Psychiatry. 1997; 36(2): 165-178.

47. Fearon RP, Bakermans-Kranenburg MJ, van IJzendoorn MH, Lapsley A-M, \& Roisman GI. The significance of insecure attachment and disorganization in the development of children's externalizing behavior: A meta-analytic study. Child Dev, 2010; 81(2), 435-456.

48. Dykas MJ, Ziv Y, Cassidy J. Attachment and peer relations in adolescence. AttachHum Dev 2008;10(2):123-41.

49. Goodman G, Stroh M, Valdez A. Do attachment representations predict depres-sion and anxiety in psychiatrically hospitalized prepubertal children? Bull Menninger Clin 2012;76(3):260 289.

50. Khan F, Fraley RC, Young JF, ve Hankin BL. Developmental trajectories of attachment and depressive symptoms in children and adolescents. Attach Hum Dev, 2019; 1-17

51. Madigan S, Brumariu LE, Villani V, Atkinson L, ve Lyons-Ruth $K$. Representational and questionnaire measures of attachment: $A$ metaanalysis of relations to child internalizing and externalizing problems. Psychol Bull, 2016; 142(4), 367-399.

52. Arguz Cildir D, Ozbek A, Topuzoglu A, Orcin E, ve Janbakhishov Evcen C. Association of prenatal attachment and early childhood emotional, behavioral, and developmental characteristics: A longitudinal study. Infant Ment Health J. 2019; 1-11

53. Punamäki RJ, Isosävi S, Qouta SR, Kuittinen S, ve Diab SY. War trauma and maternal-fetal attachment predicting maternal mental health, infant development, and dyadic interaction in Palestinian families. Attach Hum Dev, 2017; 19(5), 463- 486. 